\title{
STUDIES ON THE MECHANISM OF
}

\section{RNA SYNTHESIS OF A MURINE CORONAVIRUS}

\author{
Michael M.C. Lai, Ralph S. Baric, Peter R. Brayton, \\ and Stephen A. Stohlman \\ University of Southern California, School of Medicine \\ 2025 Zonal Avenue, Los Angeles, CA 90033
}

\section{ABSTRACT}

The mechanism of viral RNA replication in mouse hepatitis virus (MHV)-infected cells was studied by oligonucleotide mapping of every mRNA. We discovered that an oligonucleotide, No. 10, was localized at the 5'-end of every mRNA, and was not colinear with the sequences of the virion genomic RNA. This result indicates that all of the mRNAs contain a leader sequence which is joined to the body sequences of the mRNAs. We have also studied the structure of the replicative intermediate (RI) RNA in the MHV-infected cells. This RI RNA consists of a single species corresponding to the MHV genomic RNA. No subgenomic RI structures were detected. Furthermore, the nascent RNA chains in the RI structure contained the leader sequences, suggesting that the leader RNA was not added to the mRNA post-transciptionally, but rather, it was probably synthesized independently and then used as a primer for the synthesis of mRNAs. We have also shown that the poly (A) sequences in the MHV genome were transcribed from the poly (U) sequences present in the negative-strand template. The RNA polymerases involved in the MHV RNA synthesis were also characterized. The early polymerase synthesizes a single negative-stranded, full-length RNA. The late polymerases could be separated into two activities, one synthesizing positive-stranded genomic RNA, and the other synthesizing genomic as well as subgenomic RNAs. Thus, the replication and transcription functions of MHV could probably be separated. A plausible model of MHV replication is presented.

\section{INTRODUCTION}

6 Murine coronaviruses contain a single-stranded $60 \mathrm{~S}$ RNA of $5.4 \mathrm{x}$ $10^{6}$ daltons in molecular weight (Lai and Stohlman, 1978). This RNA has a 
cap structure at the 5'-end and a stretch of poly $(A)$ at the 3 '-end (Lai and Stohlman, 1981). It can be translated in vitro into several proteins in cell-free translation systems (Leibowitz et al., 1982). Therefore, the genomic RNA of murine coronaviruses is positive-stranded. The RNA genome can be divided into at least seven genetic regions (Lai et al., 1981), which encode three structural proteins, pp50, gp25, and gp90/180 (Sturman and Holmes, 1983), and potentially can also code for at least four nonstructural proteins. One or more of these nonstructural proteins could be the RNA-dependent RNA polymerases (Brayton et al., 1982; Mahy et al., 1983), which are responsible for the transcription and replication of viral RNA.

Seven virus-specific mRNA species have been detected in the murine coronavirus-infected cells (Lai et al., 1981; Leibowitz, et al. 1981; Spaan, et al., 1981). These mRNAs, ranging from $0.6-5.4 \times 10^{6}$ daltons, are arranged as a nested set from the 3'-terminus of the genome, so that the sequences of each smaller RNA are conserved at the 3'-end of every larger RNA (Lai et al., 1981). All of the virus-specific mRNAs are capped and polyadenylated, and share at least 5 nucleotides at the 5 '-ends (Lai et al., 1982a). Furthermore, some of the mRNAs contain a unique oligonucleotide which is not present in the genomic RNA (Lai et al., 1981, 1982a; Leibowitz et al., 1981). These data suggest that there might be some unusual mechanism of RNA processing involved in the synthesis of mRNAs of murine coronaviruses.

The mechanism of RNA synthesis in murine coronaviruses has only been partially elucidated. We have shown previously that these viruses replicate through a negative-sensed RNA intermediate of full genomic length (Lai et al., 1982b). Consistent with this finding, we have also shown that there is only one single species of double-stranded replicative form (RF) RNA, corresponding to the genomic-sized RNA (Lai et al., 1982b). These results suggest that all of the mRNAs are transcribed from the same genome-sized negative-stranded RNA template. The transcription of both the negative-stranded RNA template and mRNAs appear to be mediated by the virus-coded RNA-dependent RNA polymerases (Brayton et al., 1982). But the detailed mechanism of RNA synthesis is largely unknown. To understand further the replication cycle of murine coronavirus, we have studied the sequences of mRNAs, and the properities of replicative intermediates and RNA polymerases of a murine coronavirus, the A59 strain of mouse hepatitis virus (MHV).

\section{MATERIALS AND METHODS}

Virus and cells. The A59 strain of mouse hepatitis virus (MHV-A59) was used throughout this study. DBT cells or Sac (-) cells were used for the growth of virus and preparation of intracellular RNA. The procedures for the growth of virus have been described (Lai et al., 1981). All experiments were performed at $37^{\circ} \mathrm{C}$ at a multiplicity of infection of $1-5$ and in the presence of actinomycin $\mathrm{D}(2 \mathrm{ug} / \mathrm{ml})$. 
Preparation of intracellular RNA. The MHV-infected cells were labeled with ${ }^{32}$ p-orthophosphate at $6 \mathrm{~h}$ post-infection (p.i.) for various lenghts of time. The intracellular 3 NA was extracted by SDS-phenol as described (Lai et al., 1981). The ${ }^{32}$ P-labeled MHV-specific RNA was separated by electrophoresis on 1\% agarose gels and each RNA band was eluted out of the gel.

For preparation of replicative intermediate (RI) RNA, the intracellular RNA was adjusted to $2 \mathrm{M} \mathrm{NaCl}$ and $0.05 \%$ SDS, and incubated at $0-4^{\circ} \mathrm{C}$ for $24-30 \mathrm{~h}$. Afterwards, the incubation mixture was centrifuged at $14,500 \mathrm{rpm}$ in a $\mathrm{Ti} 30$ rotor for $60 \mathrm{~min}$. The precipitate was resuspended in LSB buffer (0.01 M Tris $\mathrm{HCl}, \mathrm{pH} 7.4$ and $1 \mathrm{mM}$ EDTA) containing $1 \%$ SDS and fractionated by chromatography on a Sepharose 2B-CL column $(98 \times 1.5 \mathrm{~cm})$ (Spector and Baltimore, 1975). The fractions in the void volume consisted of the replicative intermediate RNA.

Oligonucleotide fingerprinting and mapping. The ${ }^{32}$ p-labeled RNA prepared from the agarose gels or from the RI was digested exhaustively with RNase $\mathrm{T}_{1}$ and separated by two-dimensional polyacrylamide gel electrophoresis as described (Lai, et al., 1981). Briefly, the first dimension was performed on $8 \%$ polyacrylamide gel slabs $(30 \times 10 \times 0.15 \mathrm{~cm})$ at $\mathrm{pH} 3.3,700 \mathrm{~V}$ for 4 hours. The second dimension was performed on $22 \%$ polyacrylamide gel slabs $(40 \times 35 \times 0.075 \mathrm{~cm})$ at $\mathrm{pH} 8.0,650 \mathrm{~V}$ for 16 hours.

For oligonucleotide mapping, the RNA was partially degraded by boiling at $100^{\circ} \mathrm{C}$ for $3 \mathrm{~min}$. The poly (A)-containing RNA fragments were selected by chromatography on oligo (dT)-cellulose. These RNAs were separated by sucrose gradient sedimentation and each size fraction was studied by oligonucleotide fingerprinting.

Membrane fractionation and RNA polymerase assay. The MHV-infected cells were disrupted by Dounce homogenization in RSB buffer $(0.01 \mathrm{M}$ Tris $\mathrm{HCl}, \mathrm{pH} 7.4,0.01 \mathrm{M} \mathrm{NaCl}$ and $0.0015 \mathrm{M} \mathrm{MgCl}_{2}$ ). After removal of the nuclei, the cellular membranes were centrifuged in a 9 to $50 \%$ sucrose gradient made in $2 \times R S B$ with 40 ug dextran sulfate per $\mathrm{ml}$ at $90,000 \mathrm{xg}$ for $1.5 \mathrm{~h}$. The $0.2 \mathrm{ml}$ fractions were collected and each fraction was assayed for RNA polymerase activity according to published procedures (Brayton, et al., 1982).

\section{RESULTS}

Presence of Leader RNA Sequences in the mRNAs of mouse hepatitis virus (MHV). Previous studies of the sequences of the MHV mRNAs indicated that the 5'-ends of each mRNA share at least 5 nucleotides, and that several mRNAs contain a $\mathrm{T}_{1}$-oligonucleotide not present in the oligonucleotide fingerprints of the virion genomic RNA (Lai et al., 1981, 1982a). These data suggest the possible presence of leader RNA sequences in the mRNAs. To identify such RNA sequences, we studied all of the large $\mathrm{T}_{1}$-oligonucleotides in every mRNA with respect to their map 
locations on the respective mRNA. This study was designed to determine whether all of the sequençes in the mRNAs are colinear with those in the genomic RNA. The ${ }^{2}$ P-labeled mRNAs were separated by gel electrophoresis, eluted and then partially degraded by boiling. The poly (A)-containing fractions of such partially degraded mRNAs were selected by oligo (dT)-cellulose chromatography and separated by sucrose gradient sedimentation. The different size fractions separated under such conditions represent the 3'-RNA fragments of different sizes derived from each mRNA. These mRNA fragments were studied by $T_{1}$-oligonucleotide fingerprinting. Some of these fingerprints are shown in Fig. 1. Examination of these oligonucleotides showed that all of the oligonucleotides in the different mRNAs and genomic RNA are mapped at the corresponding positions, i.e. they are colinear in all of the mRNAs and genomic RNA. However, closer examination revealed an oligonucleotide, No. 10, which is not present at the same position in each mRNA species. It is located at the $5^{\prime}$-ends of every mRNA (Fig. 2). Since the oligonucleotide No. 10 is present only once in the genomic RNA, this result indicates that this oligonucleotide represents a leader RNA segment which is joined to the body sequences of every mRNA of MHV. Since this oligonucleotide has 23 bases, and the first 5 nucleotides at the 5'-ends of mRNAs are identical for all of the mRNAs (Lai et al., 1982a), the leader RNA sequences must be at least 28 nucleotides long. These results provided the strongest evidence so far for the presence of leader RNA sequences in MHV mRNAs. Thus, the unique oligonucleotides which are present only in the mRNAs could represent the junction oligonucleotides between the leader and the body sequences of mRNAs. This is consistent with the base sequences of these oligonucleotides, which showed that they are identical at the 5'-half, but differ at the 3'-half, sequences of the oligonucleotides (Lai et al., 1982a). The 5'-half presumably is derived from the leader and the 3'-half from the body sequences of the mRNAs.

Models of mRNA Synthesis in MHV. The presence of the leader RNA sequences in the MHV mRNAs suggest that RNA splicing might be involved in the synthesis of mRNA. However, UV transcriptional mapping studies suggest that the mRNAs are not derived from the cleavage of precursor RNAs (Jacobs et al., 1981). These two pieces of data indicate that the leader RNA and the body suquences of MHV mRNAs must be joined by a novel nucleus-independent mechanism. We propose three possible mechanisms for the synthesis of such mRNAs (Fig. 3): In the first model, the RNA polymerase "jumps" from the leader RNA region to various initiation sites for different mRNAs, probably as a result of "looping out" of the RNA template. The second model proposes that the leader RNA is synthesized independently and falls off the negative-stranded RNA template. This free leader RNA then binds to RNA polymerase or a short complementary region at the initiation sites for different mRNAs. In this fashion, the leader RNA serves as a primer for mRNA synthesis. The third model proposes that the leader RNA and the body sequences of MHV mRNAs are synthesized independently and then linked together post-transcriptionally by an unknown mechanism. 


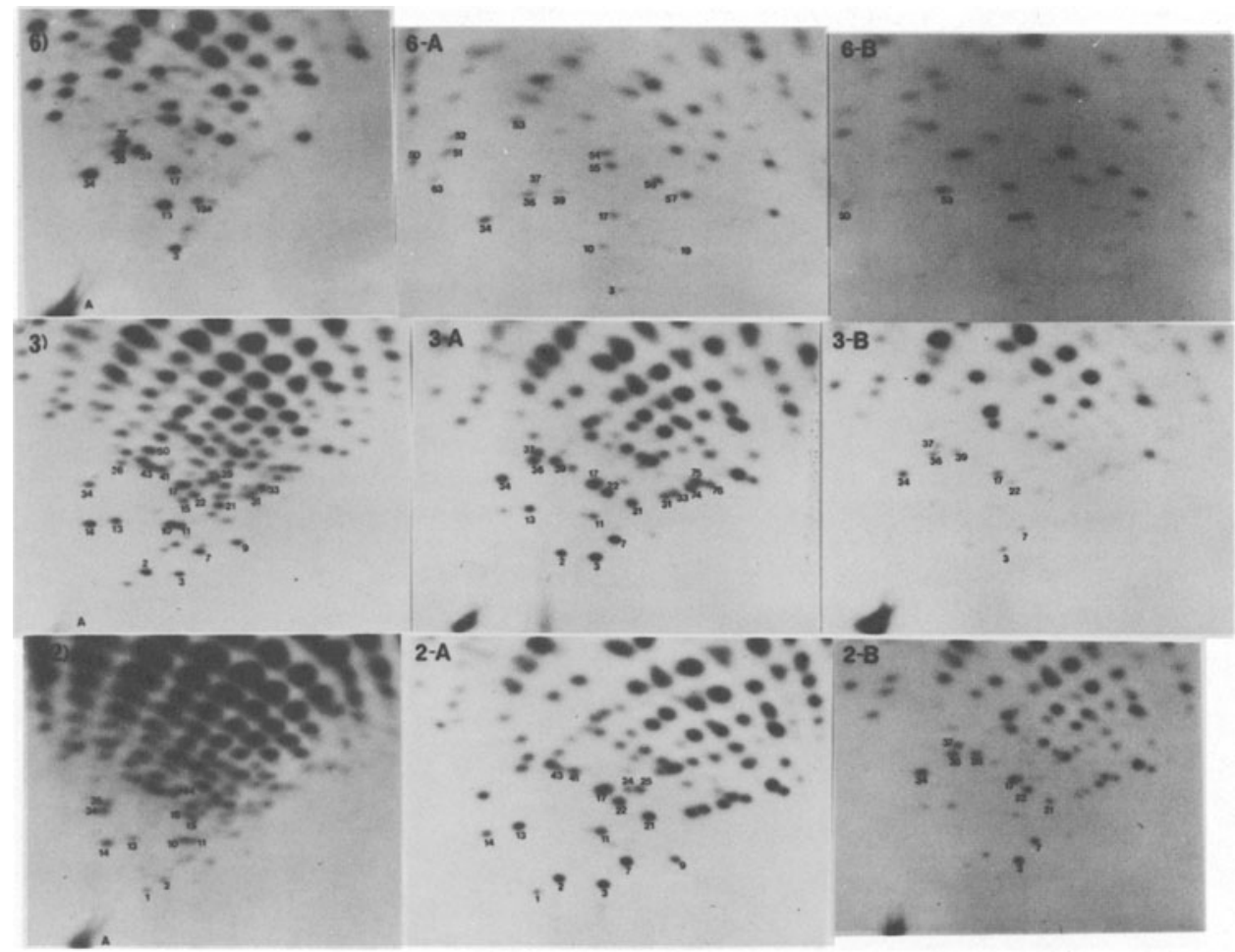

Fig. 1. Oligonucleotide maps of mRNAs \#6,3 and 2. The poly (A)containing RNA fragments derived from different mRNAs were digested with RNase $T_{1}$ and fingerprinted. (6) (3) and (2) represent full-length mRNAs. The other panels represent the fragments derived from the 3'-ends of different mRNAs. Note that the oligonucleotide No. 10 was detected only in the full-length mRNA.

$(\underline{10}, \underline{19}, 57,56,55,51,36,34,52) 50(53,54) * 7$

$(\underline{10(?)}, 19 \mathrm{a}, 3.17,37,39,63)(57,56,55,51,36,34,52) 50(53,54) * 6$

$(10(?), 3 \mathrm{a}, 75,76,74,22)(3,17,37,39,63)(57,56,55,51,36,34,52) 50(53,54) * 5$

$(\underline{10}, 43)(9,41,50,25,11,14)(21,13,2,33,31,7,75,76,74,22)(3,17,37,39,63)(57,56,55,51,36,34,52) 50(53,54) * 3$

$(\underline{10} .16,15,44,35)(24,1,43)(9,41,50,25,11,14)(21,13,2,33,31,7,75,76,74,22)(3,17,37,39,63)(57,56,55,51,36,34,52) 50(53,54) * 2$

Fig. 2. Schematic drawing of oligonucleotide maps of MHV mRNAs. The oligonucleotides were arranged in the order of $5^{\prime} 3^{\prime}$ ends. The order of the oligonucleotides within each bracket is not certain. 
MODEL 1: "LOOP OUT"
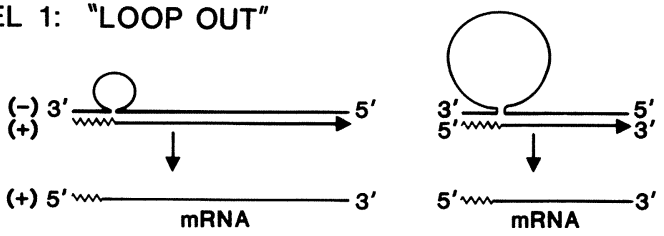

MODEL 2: LEADER-PRIMED TRANSCRIPTION<smiles>C#CCOC(=O)OCCOC</smiles>

INDEPENDENTLY

SYNTHESIZED

LEADER RNA

MODEL 3: POST-TRANSCRIPTIONAL PROCESSING

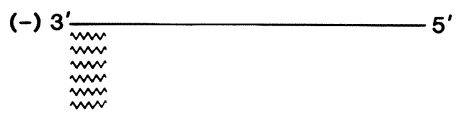

INDEPENDENTLY
SYNTHESIZED

LEADER RNA

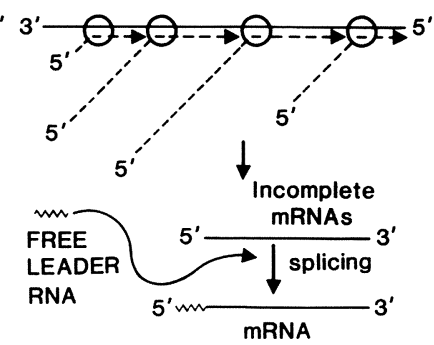

Fig. 3. Proposed models for the mechanism of transcription of MHV mRNÁs.

To distinguish these three transcriptional models for MHV, we have performed several types of experiments. The first experiment is the identification of the replicative-form (RF) RNA species derived from RNase digestion of the intracellular MHV-specific RNAs. If MHV replication utilizes the mechanism as proposed in the first model, there should be seven RF RNA species of different sizes, corresponding to the seven mRNA species. But only one RF RNA of genomic size was detected (Fig. 4). This result makes the first model quite unlikely. To decide between the second and the third model, we studied the replicative intermediate (RI) RNA, which should represent the RNA structure actively involved in MHV RNA replication. The leader RNA should be present in the RI if the second model is correct. The properties of such $\mathrm{RI}$ is described in the next section. 


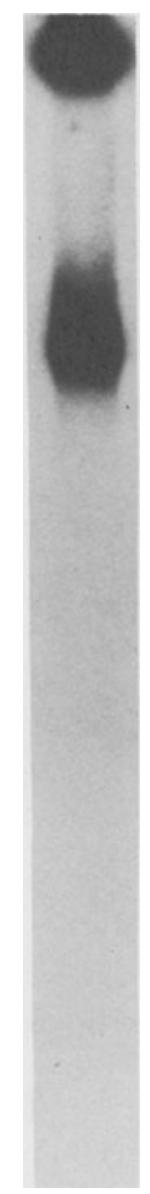

Fig. 4. Agarose gel electrophoresis of replicative form (RF) RNA. The RF RNA was isolated from the supernatant of the $2 \mathrm{M} \mathrm{NaCl}$ precipitation of the intracellular RNA followed by RNase A digestion. The electrophoresis was performed in $1 \%$ agarose gels.

Studies of Replicative Intermediate RNA of MHV. The RI RNA was prepared from the intracellular MHV-specific RNA by precipitation with $2 \mathrm{M} \mathrm{NaCl}$ followed by gel filtration chromatography on Sepharose 2B-CL. The RI RNA was collected from the void volume (the first peak in Fig 5A) and was found to be $38 \%$ RNase A-resistant. This result indicates that this RNA structure is partially double-stranded and partially single-stranded as predicted from the structure of replicative intermediates. The RI RNA was further analyzed by agarose gel electrophoresis and found to migrate as a single band with an electrophoretic mobility very closely resembling that of the single-stranded genomic RNA (Fig. 5B). The RI RNA was heat-denatured into a heterogeneous collection of RNAs ranging from the genomic size to RNAs smaller than the mRNA 非 7 . This result is consistent with the interpretation that the RI structure consists of a genome-length negative-stranded RNA, hydrogen-bonded to multiple growing nascent $(+)$-strand RNAs. RNase digestion of this RI structure produced a single species of double-stranded RF RNA (data not shown). This result again demonstrated that the mechanism depicted by the first model is not applicable to the transcription of MHV RNA. 

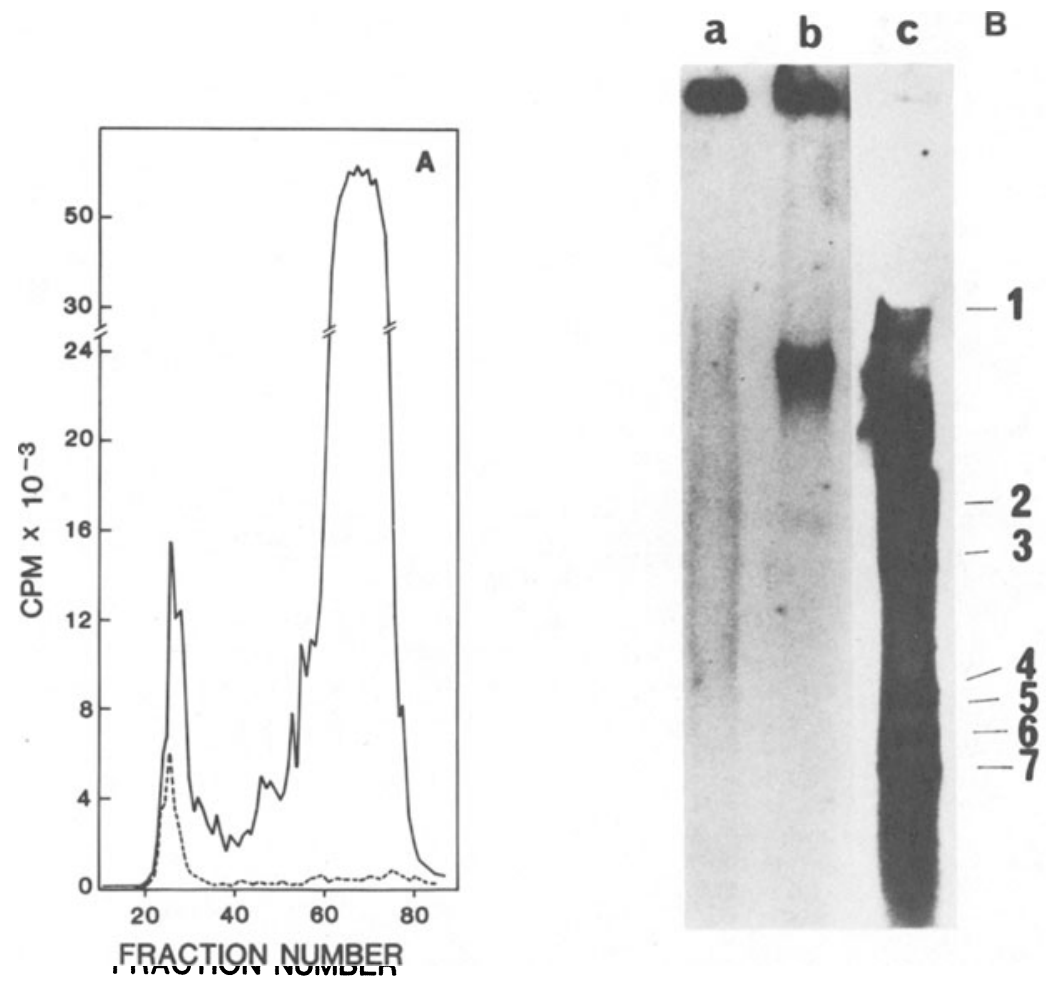

Fig. 5 Characterization of replicative intermediate (RI) RNA. Chromatography of Sepharose 2B-CL column of the $2 \mathrm{M} \mathrm{NaCl}$ precipitate of the ${ }^{32}$ P-labeled intracellular RNA. (B) Agarose gel electrophoresis of the first peak in (A). (a) heat-denatured RI; (b) native RI; and (c) total mRNAs as molecular weight markers.

To further distiguish the second from the third models of RNA transcription, we studied the oligonucleotide fingerprints of the RI RNA. If the second model is correct, the nascent $(+)$-strand RNAs are expected to contain the leader RNA sequences. On the other hand, if the third model is correct, the leader RNA sequences will not be present in the RI structure, since the leader RNA is added to the mRNA post-transcriptionally. We performed the oligonucleotide fingerprinting of the RI without heat-denaturation. This approach will identify only the newly synthesized nascent (+) RNA strands which have become the single-stranded RNA tails in the RI structure (see Fig. 9 for the possible structure of RI). As shown in Fig. 6, the "leader"-specific oligonucleotide, No. 10, was present in the RI. This result indicates that the leader RNA sequneces are joined to the mRNAs during transcription, rather than post-transcriptionally. Thus the second model is the most likely mechanism of MHV RNA transcription. 
Additional information concerning the mechanism of RNA synthesis was obtained from the oligonucleotide fingerprints of the heat-denatured RI. This reflects the sequences represented in the double-stranded portion as well as the single-stranded tails, of RI. Fig. 6B shows that the oligonucleotide fingerprint of the heat-denatured RI is essentially identical to that of the undenatured RI. However, the former contains a prominent poly (A) spot. This result suggests that the poly (A) sequences are transcribed from the poly (U) sequences present on the negative-stranded RNA templates, rather than added post-transcriptionally. This mechanism is similar to that observed during the replication of poliovirus (Spector and Baltimore, 1975).

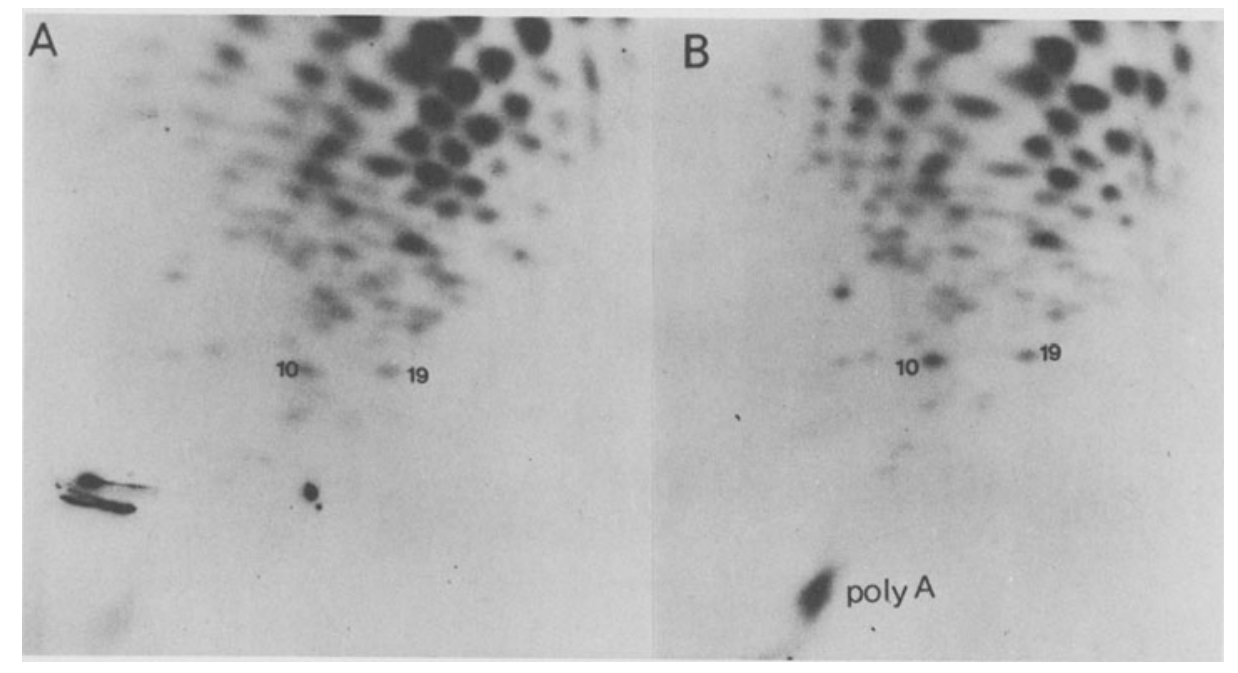

Fig. 6. Oligonucleotide fingerprints of the RI RNA. (a) native; (b) heat-denatured.

Studies of MHV-specific RNA polymerases. The studies described in the previous sections suggest that a very unique mechanism of RNA synthesis is employed in the synthesis of MHV RNA. Therefore, the RNA polymerases of MHV might possess very unique biochemical properties. Previous studies in our laboratories have indicated that two different RNA polymerases, one early ( 1 hour post-infection) and the other late ( 6 hour post-infection), can be detected in MHV-infected cells. We further studied the properties of these two polymerases in order to understand the mechanism of MHV RNA synthesis. We first attempted to fractionate the membrane complexes containing the RNA polymerase activities in MHV-infected cells. We subjected the cellular membranes, excluding the nuclei, from the MHV-infected cells to sucrose gradient sedimentation analysis. As shown in Fig. 7, the early RNA polymerase activity was contained in a single membrane complex, while the late RNA polymerase activity was divided into two separate membrane complexes. The functions of the polymerases contained in these membrane complexes 
were examined by studying the sense and size of the RNA species made by these membrane complexes in vitro. As shown in Table 1, the early RNA polymerase synthesizes RNA of negative sense which was completely hybridized by virion genomic RNA. Furthermore, the RNA synthesized consisted of a single RNA species of genomic size (Fig. 8). This is consistent with the properties of the MHV-specific RNA made in the infected cells early in the infection (Lai, et al, 1982). The RNA made in vitro by the two membrane complexes at $6 \mathrm{~h}$ p.i., on the other hand, was positive-stranded, since they were completely hybridized by the representative CDNA of the MHV genome but not the genomic RNA itself (Table 1). The heavy $(\mathrm{H})$ fraction of the late membrane complexes synthesized genomic as well as most, if not all, of the subgenomic mRNAs similar to those found in the MHV-infected cells. These results indicate that the RNA polymerase contained in this fraction is the enzyme responsible for the synthesis of mRNAs in vivo. Therefore, this membrane complex can be considered as the transcription complex. Surprisingly, the light (L) fraction of the late membrane complexes synthesized only a single species of RNA, which is of genome size. This result indicates that this fraction might be the enzyme responsible for the replication of the viral RNA genome. Therefore, this membrane complex might be a replication complex.

\section{Table 1}

Hybridization of the in vitro RNA products of the early and late polymerase peaks with MHV-A59 RNA or CDNA

The membrane fractions separated by sucrose grandients as shown in Fig. 7 were assayed for RNA polymerase activities. The RNA products obtained from the activity peaks were phenol-extracted and used for hybridization with excess (at least 100 fold) of genomic RNA or CDNA made with genomic RNA. Hybridization was perfomed in the presence of $0.75 \mathrm{M} \mathrm{NaCl}$ at $68^{\circ} \mathrm{C}$ for $12-16$ hours as described elsewhere (Lai et al., 1979). 


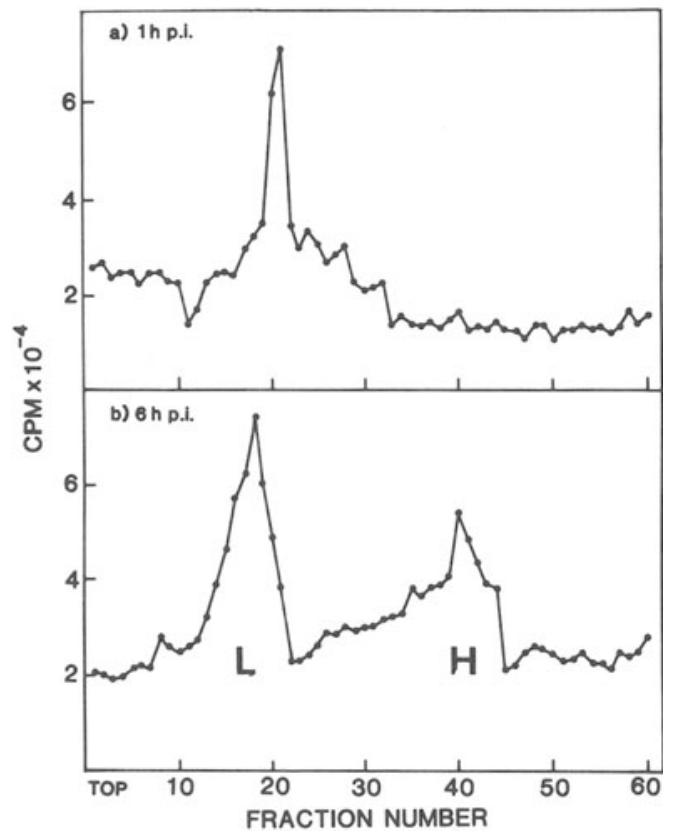

Fig. 7. Sucrose gradient sedimentation of the polymerase-containing membrane complexes. Sedimentation was performed on a 9-50\%sucrose gradient in an SW 27 rotor at $25 \mathrm{~K}$ for 1.5 hour. Each fraction was assayed for RNA polymerase activity as measured by incoporation of the ${ }^{3} \mathrm{H}$-UTP.

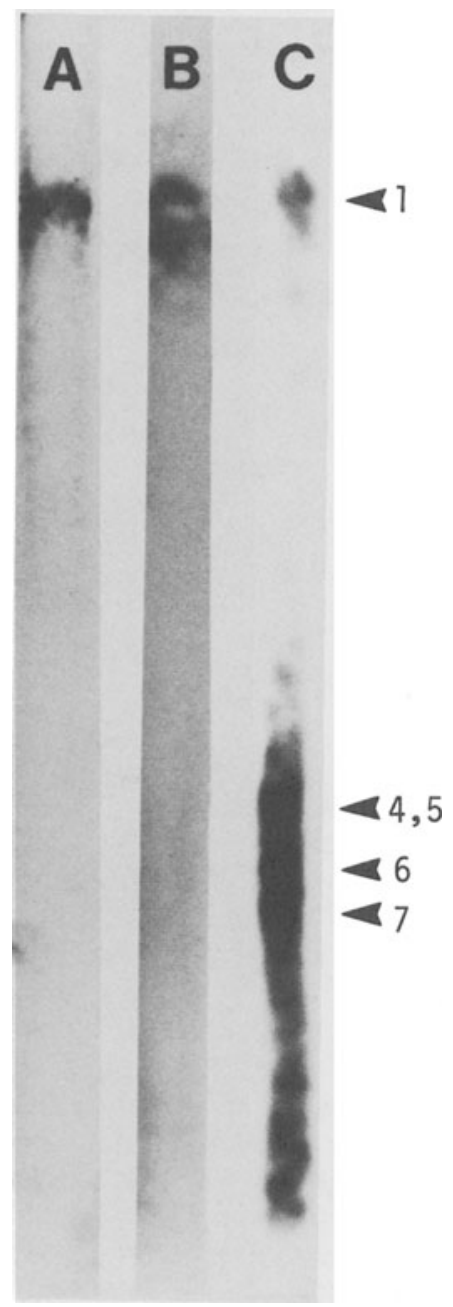

Fig. 8. Agarose gel electrophoresis of the RNA products of the in vitro RNA polymerase activities. (a) 1 hr p.i.; (b) 6 hr. p.i. L fraction; and (c) $6 \mathrm{hr}$. p.i. $\mathrm{H}$ fraction. 


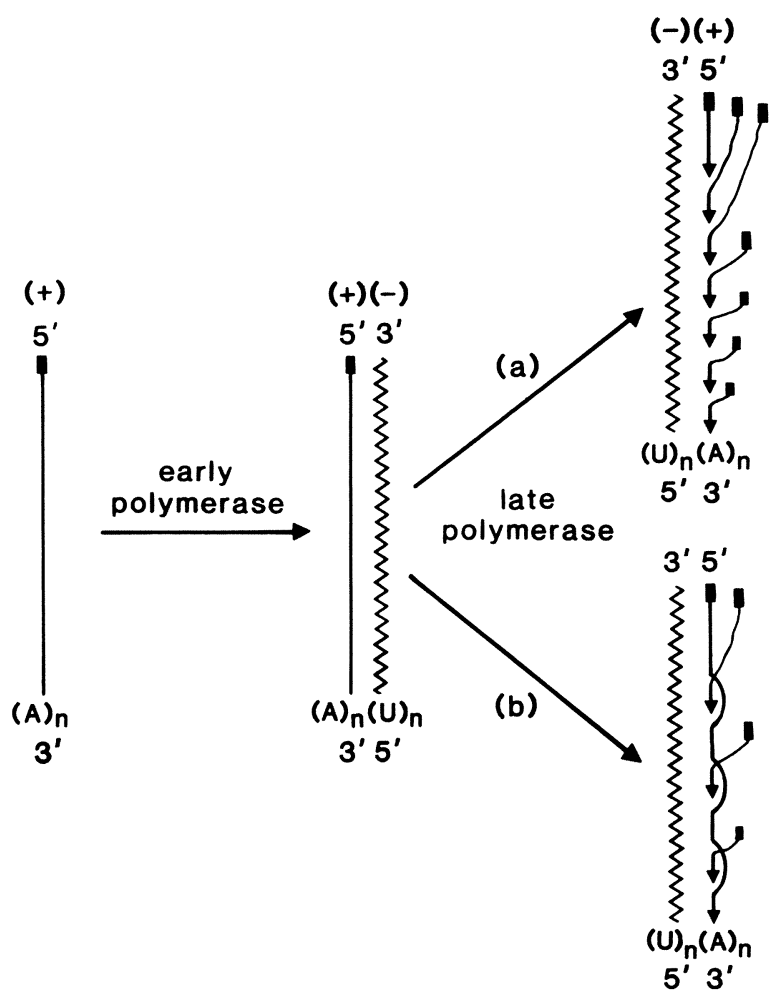

Fig. 9. $\quad$ Proposed models of the replication of MHV. The solid boxes at the ends of the (t) strands represent the leader sequences. semiconservation; (b) conservation mechanisms.

\section{DISCUSSION}

From the studies published elsewhere (Lai et al., 1982a,b; Brayton et al., 1982; Jacobs et al., 1981) and presented in this communication, the replication cycle of murine coronaviruses can be summarized as in Fig. 9. It is clear that the RNA synthesis, particularly the mRNA synthesis, of murine coronaviruses involves a very complex and unique mechanism. It involves the fusion of two noncontiguous RNA segments, one of which serves as the leader or primer for the synthesis of every mRNA species. It appears that all of the mRNAs contain the same kind of leader RNA sequences. These leader sequences are also present at the 5'-end of the virion genomic RNA, and, therefore, might be transcribed from the 3'-end of the negative-stranded full-length RNA intermediate. The mechanisms by which the leader RNA is synthesized and joined to the body sequences of the mRNAs are still poorly understood. But, it is most likely, as 
proposed by the model 2 (Fig. 3) that the leader RNA is synthesized independently and then serves as the primer for the synthesis of the body sequences of mRNAs. Therefore, this process is entirely different from the conventional post-transcriptional RNA splicing. It is, in some aspects, similar to the RNA synthesis of influenza virus (Krug, 1981). But, in the case of coronavirus, the primer RNA is more likely viral RNA rather than cellular mRNA. The presence of leader RNA is also reminiscent of vesicular stomatitis virus (Colonno and Banerjee, 1976). But, these two viruses are again different, since the leader RNA in VSV is not joined to the mRNAs. Therefore, the mechanism of mRNA synthesis in MHV is unique among all of animal viruses.

There are still many unanswered and puzzling questions in the mechanism of RNA synthesis for murine coronaviruses: First of all, the mechanism by which the leader RNA is synthesized and then used as a primer is unknown. So far, no free leader RNA has been detected in the MHV-infected cells. Secondly, the structure of the replicative intermediate poses a very difficult problem. This RI must be simultaneously synthesizing 7 mRNAs at different rates. The entire sequences of every negative-strand RNA template probably are used for transcription. Furthermore, there must be a mechanism by which the rate of initiation for different mRNAs are regulated. The exact details of the structure of this kind of RI remains to be studied.

The RNA polymerases involved in the synthesis of the negative-stranded RNA template early in the infection and the positive-stranded RNA later in the infection are probably different (Brayton et al, 1982). However, it is not clear whether they are two different enzymes or a modification of the same enzyme. If the latter is the case, modification could be caused by a viral gene product of by a host cell factor. It is not yet possible to distinguish between these two possibilities.

The possible separation of the transcription and replication complexes raises an interesting question. Is there a structural difference between the genomic RNA destined for packaging into viral particles and that used for mRNAs? Such a difference could conceivably be achieved by the way the leader RNA is joined to the viral RNA. Such issue requires further studies.

\section{ACKNOWLEDGMENT}

We thank Chris Patton, Todd Kennell and Mahmood Kafaii for excellent technical assistance, and Cathy Wung, Raymond Mitchell and Alisa Young for editorial assistance in manuscript preparation.

This work was supported by Public Health Service research grant AI 19244 from the National Institutes of Health, grant PCM-4507 from the National Science Foundation, and grant RG 1449-A from the National Multiple Sclerosis Society. 


\section{REFERENCES}

- Brayton, P.R., Lai, M.M.C., Patton, C.D and Stohlman, S.A. 1982. Characterization of two RNA polymerase activities induced by mouse hepatitis virus. J. Virol. 42:847-853.

- Colonno, R.J. and A.K. Banerjee. 1976. A unique RNA species involved in initiation of vesicular stomatitis virus RNA transcription. Cell 8:197204.

- Jacobs, L., W.J.M. Spaan, M.C. Hor zinek and B.A.M. Van der Zeijst. 1981. Synthesis of subgenomic mRNAs of mouse hepatitis virus is initiated independently:evidence from UV transcription mapping. J. Virol. 39:401406.

- Krug, R.M. 1981. Priming of influenza viral RNA transcription by capped heterologous RNAs. Curr. Topics in Microb. Immunol. 93:125-149.

- Lai, M.M.C., Brayton, P.R., Armen, R.C., Patton, C.D., Pugh, C., and S.A. Stohlman. 1981. Mouse hepatitis virus A59: Messenger RNA structure and genetic localization of the sequence divergence from a hepatotropic strain MHV-3. J. Virol. 39:823-834.

- Lai, M.M.C., S.S.F. Hu and P.K. Vogt. 1979. Avian erythroblastosis virus: Transformation-specific sequences form a contiguous segment of $3.25 \mathrm{~kb}$ located in the middle of the 6-kb genome. Virology 97:366-377.

- Lai, M.M.C., Patton, C.D., and S.A. Stohlman. 1982a. Further characterization of mRNAs of mouse hepatitis virus: presence of common 5'-end nucleotides. J. Virol. 41:557-565.

- Lai, M.M.C., C.D. Patton and S.A. Stohlman. 1982b. Replication of mouse hepatitis virus: negative-stranded RNA and replicative form RNA are of genome length. J. Virol. 44:487-492.

- Lai, M.M.C. and S.A. Stohlman. 1978. The RNA of mouse hepatitis virus. J. Virol. 271:236-242.

- Lai, M.M.C. and Stohlman, S.A. 1981. Comparative analysis of RNA genome of mouse hepatitis virus. J. Virol. 38:661-670.

- Leibowitz, J.L., S.R. Weiss, E. Paavola and C.L. Bond. 1982. Cell-free translation of murine coronavirus RNA. J. Virol. 43:905-913.

- Leibowitz, J.L., K.C. Wilhelmsen and C.W. Bond. 1981. The virusspecific intracellular RNA species of two murine coronaviruses: MHVA59 and MHV-JHM. Virology 114:29-51.

- Mahy, B.W.J., S. Siddell, H. Wege and V. ter Meulen. 1983. RNAdependent RNA polymerase activity in murine coronavirus-infected cell. J. Gen. Virol. 64:103-111.

- Spaan, W.J.M., P.J.M. Rottier, M.C. Horzinek and B.A.M. van der Zeijst. 1981. Isolation and identification of virus-specific mRNAs in cells infected with mouse hepatitis virus (MHV-A59). Virology 108:424-434.

- Spector, D.H. and D. Baltimore. 1975. Polyadenylic acid on poliovirus RNA. II. Poly A on intracellular RNAs. J. Virol. 15:1418-1431.

- Sturman, L.S. and K.V. Homes. 1983. The molecular biology of coronaviruses. Adv. in Virus Research (in press). 